\title{
Synthesis and Reactions of New 4-Oxo-4H-benzopyran-3-carboxaldehydes Containing Hydroxy Groups or 2-Oxopyran Cycles
}

\author{
Margita Lacova*1, Dusan Loos, Mikulas Furdik ${ }^{1}$, Maria Matulova ${ }^{2}$ and Hafez M. El-Shaaer ${ }^{3}$ \\ 1 Department of Organic Chemistry, Faculty of Natural Sciences, Comenius University, SK-84215 Bratislava, Slovak \\ Republic. Tel. +42107 60296338, Fax +4210765429064 (lacova@fns.uniba.sk) \\ 2 Slovak Academy of Sciences, 84238 Bratislava, Slovak Republic \\ 3 Department of Chemistry, Faculty of Education,Ain Shams University, Roxy, Cairo, Egypt
}

\begin{abstract}
The synthesis of eight hydroxy- and 2-oxopyranochromone-3-carboxaldehydes 3, 5 and their reactions with 2-hydroxyaniline, 2,4-dinitrophenylhydrazine and 2-benzothiazolylhydrazine were investigated. Products were confirmed by IR, NMR spectral and elemental analysis data. The semi-empirical AM1 quantum-chemical method has been used to study optimal geometries and heats of formation of synthesized 3formylchromones
\end{abstract}

Keywords: 3-Formylchromones, Vilsmeier - Haack reaction, 2-oxobenzopyrane, imines, enamines, AM1 calculations

\section{Introduction}

This work was done in connection with our study of synthetic, theoretical, spectral [1 - 5] and biological [6, 7] properties of 3-formylchromone derivatives. In the course of biological investigation of 3-formylchromone derivatives we found a hereditary bleaching effect on the plastid system of Euglena gracilis [7] and antimycobacterial activity similar to effect of isonicotin acid hydrazide (INH) [5, 7]. Due to their biological activity are chromone derivatives are a subject of considerable pharmaceutical and chemical interest. The natural chromones of the abundant flavonoid family contain prevailingly one or several hydroxyl groups which can be free or protected. 3-Formylchromones are also attractive syntons for preparative organic chemistry due to a behaviour similar to $\alpha, \beta$-unsaturated aldehydes [8, 9]. Therefore our attention was aimed at the investigation of favourable conditions for the preparation of two biologically interesting groups of aldehydes e.g. 3formylchromones containing the condensed 2-oxopyran

\footnotetext{
* To whom correspondence should be addressed.
}

C 1998 MDPI. All rights reserved. Molecules http://www.mdpi.org/molecules/ 
ring $\mathbf{5 a}$ - 5e and difficultly accessible aldehydes with non protected hydroxy groups at the benzene ring $\mathbf{3 a} \mathbf{a} \mathbf{- 3 c}$.

\section{Results and Discussion}

In the first part of the work the preparation of 7 hydroxy-, 6-n-hexyl-7-hydroxy- and 7, 8-dihydroxy-3formylchromones 3a - 3e was studied. It has been found that their preparation using the Vilsmeier-Haack formylation of appropriate o-hydroxyacetphenones afforded very low yields $(20-30 \%)$. Our efforts to prepare 5,7-dihydroxy-3-formylchromones by direct formylation of 2, 4, 6-trihydroxyacetphenone 1d were unsuccesfull. The reaction resulted in polymeric products in all experiments. It can be assumed that the hydroxy groups of compounds 1a - 1d caused the lowering of the acetyl group acidity and preferably enables the formylation of the benzene ring and polycondensation of intermediates. The new 2, 4-dihydroxy-5-hexylacetophenone 1c was prepared by acetylation in acetic acid and $\mathrm{ZnCl}_{2}$ at reflux in $56 \%$ yield.

In the second part of this work we developed the method of synthesis of a 3-formyl- chromone having a condensed 2-oxopyrane ring. The synthetic strategy of 3formylchromones 5a - 5e had to be based on building up the 2-benzopyrone skeleton. The key - step in this synthesis was the preparation of a suitable acetyl derivative 4a - 4d, from which the requested 3-formylchromones were obtained by Vilsmeier-Haack double formylation in $80-90 \%$ yields. The synthesis of $\mathbf{5 a}-\mathbf{5 e}$ is shown in Scheme 2.

The Vilsmeier-Haack formylation was used to afford two different aldehydes 5d and $\mathbf{5 d}_{\mathbf{1}}$ from 2-oxo-2H-6acetyl-5,7-dihydroxy-4-methylbenzopyran 4d. However, only one product was isolated from the reaction mixture. The ${ }^{1} \mathrm{H}$ NMR spectra confirmed the structure of $\mathbf{5 d}$. The signal of the proton of the hydroxy group was a singlet and a coupled constant ${ }^{4} \mathrm{~J}$ for a hydroxy group was absent.

8-Acetyl-7-hydroxy-4-methylcoumarin $\quad \mathbf{4 a}$ was prepared from 1,3-dihydroxybenzene in three reaction steps, namely by the Pechmann reaction, acetylation, and then by Fries rearrangement. All three reaction steps proceeded in high yields $(84-90 \%)$. After recrystallisation of the Fries rearrangement product another isomer $\mathbf{4 b}(6 \%)$ was isolated from the mother liquor. The product 4b (6-acetyl-7-hydroxycoumarin) was obtained directly as the main product from 2, 4dihydroxyacetophenone 1a by the Pechmann reaction in the presence of $\mathrm{POCl}_{3}$.

6-Acetyl-5-hydroxy-4-methyl coumarin $\mathbf{4 c}$ was also prepared from compound 1a by Pechmann reaction in the presence of $\mathrm{AlCl}_{3}$. 2, 4, 6-Trihydroxyacetophenone 1d yielded a mixture of both isomers $\mathbf{4 d}$ and $\mathbf{4 e}$ by Pechmann reaction in a ratio $1: 1$. The pure products $\mathbf{4 d}$ were isolated by recrystallization from ethanol. Product $\mathbf{4 e}$ was soluble and was isolated after evaporation of the mother liquor. The preparation of compounds $\mathbf{5} \mathbf{d}$ and $\mathbf{5 e}$ from the parent phenol involved three steps. Two steps of the synthesis yielded about $80-90 \%$ of products. Only the second step, the product of the Pechmann reaction gave $40-50 \%$ yield. The elemental analysis data of the prepared compounds is listed in Table 1.

The assumed structures of the aldehydes $\mathbf{3}, \mathbf{5}$ and the compounds 4 were proved by infrared and ${ }^{1} \mathrm{H}$ NMR spectra. The infrared spectra of 3-formylchromones $\mathbf{3}$ showed two strong absorption bands of the $\mathrm{C}=\mathrm{O}$ stretching vibrations belonging to the carbonyl group of $\gamma$-pyrone at $1620 \mathrm{~cm}^{-1}$ and to the aldehyde carbonyl group at 1695 $\mathrm{cm}^{-1}$.

The $\mathrm{C}=\mathrm{O}$ stretching vibrations of the carbonyl groups of 5 exhibited strong absorption bands in three very well distinguished regions: $1655-1637 \mathrm{~cm}^{-1}, 1704-1694 \mathrm{~cm}^{-1}$ and $1760-1724 \mathrm{~cm}^{-1}$ belonging to the $v(\mathrm{C}=\mathrm{O})$ of the $\gamma$ pyrone ring, the aldehyde groups and the $\alpha$-pyrone ring, respectively (Table 2 ).

The structure of the prepared compounds was also confirmed by ${ }^{1} \mathrm{H}$ NMR spectra. The resonance signals and their multiplicity are given in Table 3. In this table also included are the chemical shifts for the acetyl derivatives 4a - 4c, because these compounds were previously reported without ${ }^{1} \mathrm{H}$ NMR spectral data.

The condensation reactions of the aldehydes $\mathbf{3 a}-\mathbf{3 c}$ and $5 \mathbf{a}-\mathbf{5 e}$ were carried out with 2-hydroxyaniline, 2,4dinitrophenylhydrazine, 2-benzothiazolylhydrazine and ethyl acetoacetate. 2,4-Dinitrophenylhydrazones and 2benzothiazolylhydrazones $\mathbf{7 a}-\mathbf{7 k}$ were formed by refluxing the starting mixture in ethanol. The products appeared as coloured and slightly soluble compounds decomposing near their melting points. The reaction of 2hydroxyaniline with 3-formylchromones gives chromanones $\mathbf{8}$ or $\mathbf{9}$ using different reaction media (Scheme 3). In ethanol the adducts 8 were obtained, in diethylether the compounds 9 were formed with two molecules of 2-hydroxyaniline. The aldol condensation product $\mathbf{6}$ was obtained by heating the aldehyde 3a, and ethyl acetatoacetate with $\mathrm{CH}_{3} \mathrm{COOK}$ as catalyst.

The starting compounds $\mathbf{1}$, and 3-formylchromone derivatives 3a - 3c, 5a - 5e were studied by the semiempirical quantum chemical AM1 method [10]. The full optimisation of the geometry of every structural parameter for several conformers was performed. Heats of formation were calculated for all s-cis and s-trans conformations. The s-cis conformations appeared to be energetically more favourable then the s-trans ones. The difference in the heats of formation is about $20 \mathrm{~kJ} \mathrm{~mol}^{-1}$ for acetophenones 1 and $22-26 \mathrm{~kJ} \mathrm{~mol}^{-1}$ for 3-formylchromones $\mathbf{3}, \mathbf{5}$. In accordance with the ${ }^{1} \mathrm{H}$ NMR spectra, the results of theoretical calculation of both isomers of aldehydes $\mathbf{5 d}$ and $\mathbf{5} \mathbf{d}_{\mathbf{1}}$ (Scheme 2) shows that the isomer $\mathbf{5 d}$ is about 4.5 $\mathrm{kJ} / \mathrm{mol}$ more stable than the isomer $\mathbf{5} \mathbf{d}_{\mathbf{1}}$. 
Table 1. Elemental analysis data of prepared compounds.

\begin{tabular}{|c|c|c|c|c|c|}
\hline \multirow[t]{2}{*}{ Compound } & \multirow[t]{2}{*}{$\begin{array}{l}\text { Formula } \\
\mathrm{M}_{\mathrm{r}}\end{array}$} & \multicolumn{3}{|c|}{$\begin{array}{l}\mathrm{W}_{\mathrm{i}} \text { (calc.) } \% \\
\mathrm{~W}_{\mathrm{i}} \text { (found) \% }\end{array}$} & \multirow[t]{2}{*}{ M.p. $\left({ }^{\circ} \mathrm{C}\right)$} \\
\hline & & $\mathrm{C}$ & $\mathrm{H}$ & $\mathrm{N}$ & \\
\hline $1 \mathrm{c}$ & $\mathrm{C}_{14} \mathrm{H}_{20} \mathrm{O}_{3}$ & 71.18 & 8.51 & & $75-77$ \\
\hline & & & & & \\
\hline \multirow[t]{2}{*}{ 3a } & $\mathrm{C}_{10} \mathrm{H}_{6} \mathrm{O}_{4}$ & 63.14 & 3.17 & & $268-270$ \\
\hline & 190.2 & 63.31 & 3.10 & & \\
\hline \multirow[t]{2}{*}{$\mathbf{3 b}$} & $\mathrm{C}_{10} \mathrm{H}_{6} \mathrm{O}_{5}$ & 58.30 & 2.91 & & $264-266$ \\
\hline & 206.2 & 58.26 & 2.98 & & \\
\hline \multirow[t]{2}{*}{$3 c$} & $\mathrm{C}_{16} \mathrm{H}_{18}$ & 70.07 & 6.57 & & $233-234$ \\
\hline & 274.2 & 70.01 & 6.60 & & \\
\hline \multirow[t]{2}{*}{$5 a$} & $\mathrm{C}_{14} \mathrm{H}_{8} \mathrm{O}_{5}$ & 65.62 & 3.13 & & $310-312$ \\
\hline & 256.2 & 65.33 & 3.12 & & \\
\hline \multirow[t]{2}{*}{$5 \mathbf{b}$} & $\mathrm{C}_{14} \mathrm{H}_{8} \mathrm{O}_{5}$ & 65.62 & 3.13 & & $255-260$ \\
\hline & 256.2 & 65.48 & 3.01 & & \\
\hline \multirow[t]{2}{*}{$5 c$} & $\mathrm{C}_{14} \mathrm{H}_{8} \mathrm{O}$ & 65.62 & 6.57 & & $233-234$ \\
\hline & 256.2 & 65.32 & 3.07 & & \\
\hline \multirow[t]{2}{*}{$5 d$} & $\mathrm{C}_{14} \mathrm{H}_{8} \mathrm{O}_{6}$ & 61.79 & 2.94 & & $273-274$ \\
\hline & 272.2 & 61.62 & 2.99 & & \\
\hline \multirow[t]{2}{*}{$5 e$} & $\mathrm{C}_{14} \mathrm{H}_{8} \mathrm{O}_{6}$ & 61.79 & 2.94 & & $291-293$ \\
\hline & 272.2 & 61.77 & 2.92 & & \\
\hline \multirow[t]{2}{*}{$7 a$} & $\mathrm{C}_{17} \mathrm{H}_{11} \mathrm{~N}_{3} \mathrm{O}_{3} \mathrm{~S}$ & 60.53 & 3.26 & 12.46 & $248-250$ \\
\hline & 337.3 & 60.37 & 3.25 & 12.27 & \\
\hline \multirow[t]{2}{*}{$7 b$} & $\mathrm{C}_{23} \mathrm{H}_{23} \mathrm{~N}_{3} \mathrm{O}_{3} \mathrm{~S}$ & 65.60 & 5.46 & 9.97 & $219-220$ \\
\hline & 421.4 & 65.35 & 5.33 & 9.54 & \\
\hline \multirow[t]{2}{*}{$7 \mathrm{c}$} & $\mathrm{C}_{17} \mathrm{H}_{11} \mathrm{~N}_{3} \mathrm{O}_{4} \mathrm{~S}$ & 57.79 & 3.12 & 11.90 & $259-261$ \\
\hline & 403.3 & 57.48 & 3.11 & 11.76 & \\
\hline \multirow[t]{2}{*}{$7 d$} & $\mathrm{C}_{21} \mathrm{H}_{13} \mathrm{~N}_{3} \mathrm{O}_{4} \mathrm{~S}$ & 62.50 & 3.24 & 10.41 & $253-255$ \\
\hline & 403.3 & 62.37 & 3.23 & 10.29 & \\
\hline
\end{tabular}


Table 1. Continued.

\begin{tabular}{|c|c|c|c|c|c|}
\hline \multirow[t]{2}{*}{ Compound } & \multirow[t]{2}{*}{$\begin{array}{l}\text { Formula } \\
\mathrm{M}_{\mathrm{r}}\end{array}$} & \multicolumn{3}{|c|}{$\begin{array}{l}\mathrm{W}_{\mathrm{i}} \text { (calc.) } \% \\
\mathrm{~W}_{\mathrm{i}} \text { (found) } \%\end{array}$} & \multirow[t]{2}{*}{ M.p. $\left({ }^{\circ} \mathrm{C}\right)$} \\
\hline & & $\mathrm{C}$ & $\mathrm{H}$ & $\mathrm{N}$ & \\
\hline \multirow[t]{2}{*}{$7 e$} & $\mathrm{C}_{21} \mathrm{H}_{13} \mathrm{~N}_{3} \mathrm{O}_{5} \mathrm{~S}$ & 60.13 & 3.12 & 10.01 & $325-8$ \\
\hline & 419.3 & 60.22 & 3.19 & 9.71 & \\
\hline \multirow[t]{2}{*}{$7 f$} & $\mathrm{C}_{21} \mathrm{H}_{13} \mathrm{~N}_{3} \mathrm{O}_{4} \mathrm{~S}$ & 62.50 & 3.24 & 10.41 & $240-242$ \\
\hline & 403.3 & 62.38 & 3.20 & 10.39 & \\
\hline \multirow[t]{2}{*}{$7 \mathrm{~g}$} & $\mathrm{C}_{16} \mathrm{H}_{10} \mathrm{O}_{7} \mathrm{~N}_{4}$ & 51.90 & 2.72 & 15.13 & $297-9$ \\
\hline & 378.3 & 51.62 & 2.76 & 14.89 & decomp. \\
\hline \multirow[t]{2}{*}{$7 \mathrm{~h}$} & $\mathrm{C}_{22} \mathrm{H}_{22} \mathrm{O}_{7} \mathrm{~N}_{4}$ & 58.15 & 4.88 & 12.33 & $296-8$ \\
\hline & 454.4 & 57.86 & 4.84 & 12.09 & decomp. \\
\hline \multirow[t]{2}{*}{$7 \mathbf{i}$} & $\mathrm{C}_{16} \mathrm{H}_{10} \mathrm{O}_{8} \mathrm{~N}_{4}$ & 49.75 & 2.61 & 14.50 & $173-6$ \\
\hline & 386.3 & 49.36 & 2.66 & 14.28 & decomp. \\
\hline \multirow[t]{2}{*}{$7 \mathbf{j}$} & $\mathrm{C}_{20} \mathrm{H}_{12} \mathrm{O}_{8} \mathrm{~N}_{4}$ & 55.05 & 2.77 & 12.84 & $289-94$ \\
\hline & 436.3 & 54.89 & 2.77 & 12.75 & \\
\hline \multirow[t]{2}{*}{$7 \mathbf{k}$} & $\mathrm{C}_{20} \mathrm{H}_{12} \mathrm{O}_{9} \mathrm{~N}_{4}$ & 53.11 & 2.67 & 12.38 & $300-2$ \\
\hline & 452.3 & 52.84 & 2.80 & 12.06 & decomp. \\
\hline \multirow[t]{2}{*}{$8 \mathbf{a}$} & $\mathrm{C}_{22} \mathrm{H}_{19} \mathrm{NO}_{6}$ & 67.18 & 4.83 & 3.56 & $275-6$ \\
\hline & 393.4 & 66.89 & 4.59 & 3.12 & \\
\hline \multirow[t]{2}{*}{$8 b$} & $\mathrm{C}_{22} \mathrm{H}_{19} \mathrm{NO}_{7}$ & 64.55 & 4.65 & 3.42 & $259-60$ \\
\hline & 409.4 & 64.36 & 4.00 & 3.30 & \\
\hline \multirow[t]{2}{*}{$9 a$} & $\mathrm{C}_{26} \mathrm{H}_{20} \mathrm{~N}_{2} \mathrm{O}_{6}$ & 68.42 & 4.39 & 6.13 & $180-5$ \\
\hline & 456.4 & 68.22 & 4.51 & 6.02 & \\
\hline \multirow[t]{2}{*}{$9 b$} & $\mathrm{C}_{26} \mathrm{H}_{20} \mathrm{~N}_{2} \mathrm{O}_{6}$ & 66.10 & 4.24 & 5.92 & $158-62$ \\
\hline & 472.4 & 66.05 & 4.24 & 5.74 & \\
\hline \multirow[t]{2}{*}{$9 c$} & $\mathrm{C}_{26} \mathrm{H}_{20} \mathrm{~N}_{2} \mathrm{O}_{6}$ & 68.42 & 4.39 & 6.13 & $188-90$ \\
\hline & 456.4 & 68.51 & 4.37 & 6.19 & \\
\hline
\end{tabular}


Table 2. IR - spectral data (in $\mathrm{cm}^{-1}$ ).

\begin{tabular}{|c|c|c|c|c|c|}
\hline Compound & $\mathrm{C}^{4}=\mathrm{O}^{\mathrm{a}}$ & $\mathrm{CH}=\mathrm{O}$ & $\mathrm{C}^{8}=\mathrm{O}^{\mathrm{a}}$ & $v_{s}\left(\mathrm{NO}_{2}\right)$ & $v_{a s}\left(\mathrm{NO}_{2}\right)$ \\
\hline 3a & 1620 & 1695 & - & - & - \\
\hline $3 b$ & 1630 & 1682 & - & - & - \\
\hline $3 c$ & 1630 & 1696 & - & - & - \\
\hline $5 \mathbf{a}$ & 1657 & 1700 & 1726 & - & - \\
\hline $5 b$ & 1655 & 1693 & 1748 & - & - \\
\hline $5 c$ & 1637 & 1693 & 1700 & - & - \\
\hline $5 d$ & 1640 & 1702 & 1734 & - & - \\
\hline $5 e$ & 1640 & 1704 & 1724 & - & - \\
\hline $7 a$ & 1634 & - & - & - & - \\
\hline $7 b$ & 1630 & - & - & - & - \\
\hline $7 d$ & 1630 & - & 1720 & - & - \\
\hline $7 \mathrm{~g}$ & 1640 & - & - & 1318 & 1580 \\
\hline $7 \mathrm{~h}$ & 1612 & - & - & 1350 & 1580 \\
\hline $7 \mathbf{i}$ & 1610 & - & - & 1345 & 1580 \\
\hline $7 \mathbf{j}$ & 1640 & - & 1722 & 1345 & 1580 \\
\hline $7 k$ & 1606 & - & 1748 & 1310 & 1580 \\
\hline $8 a$ & 1642 & - & 1718 & - & - \\
\hline $8 b$ & 1642 & - & 1708 & - & - \\
\hline $9 a$ & 1648 & - & 1700 & - & - \\
\hline
\end{tabular}

${ }^{\mathrm{a}}$ For numbering of carbon atoms see Scheme 2. 


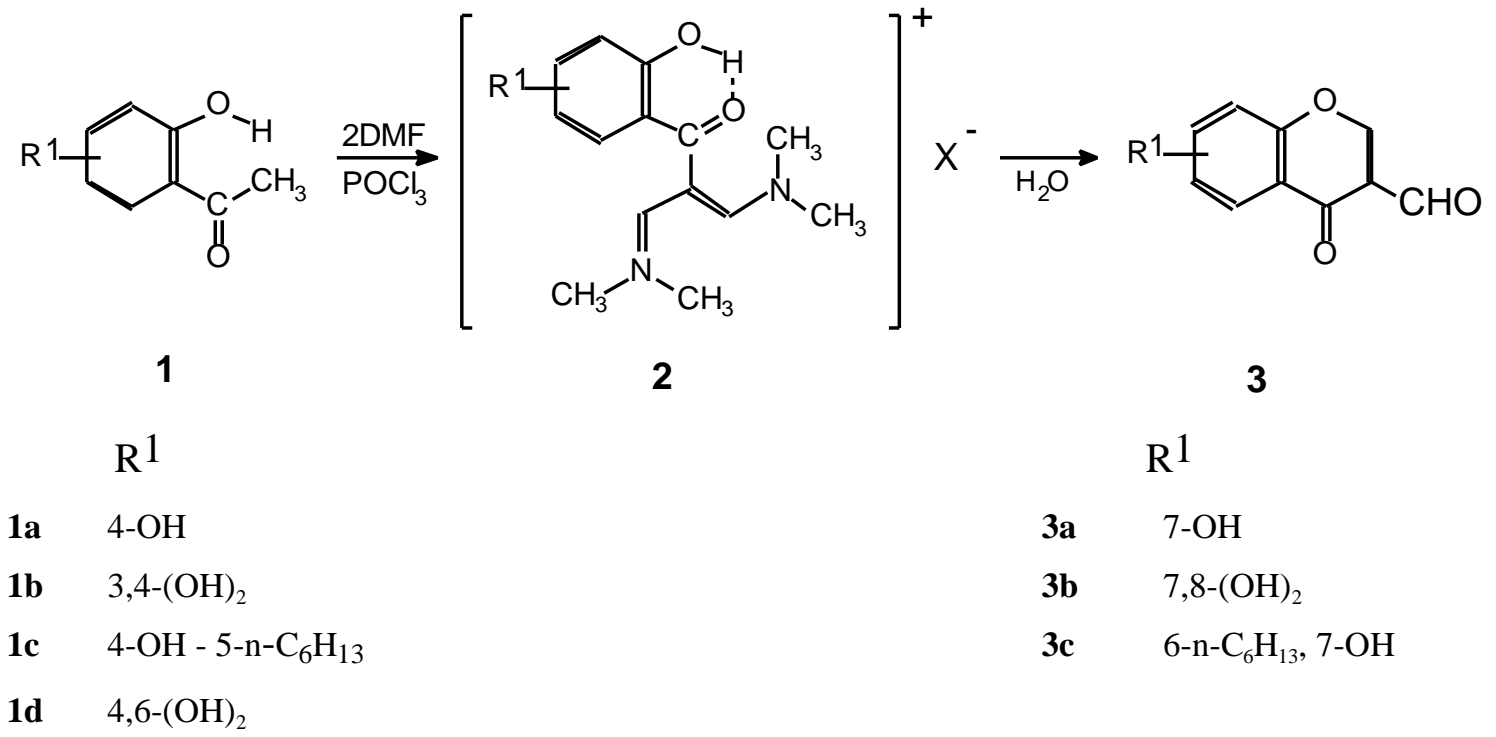

Scheme 1. 
<smiles>CC1=CC(=O)OC2C=C(OC(C)C)C=CC12</smiles><smiles></smiles><smiles>C1C[Te]C1</smiles>

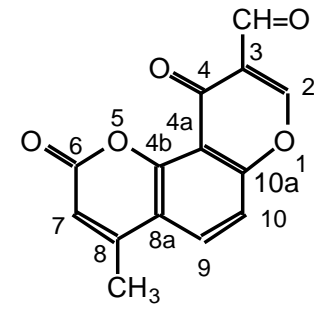

$5 a$<smiles>COc1cc2c(C)cc(=O)oc2cc1OC(=O)OC(F)(F)C(=O)O</smiles><smiles>COc1ccc2oc(=O)cc(C)c2c1O</smiles>

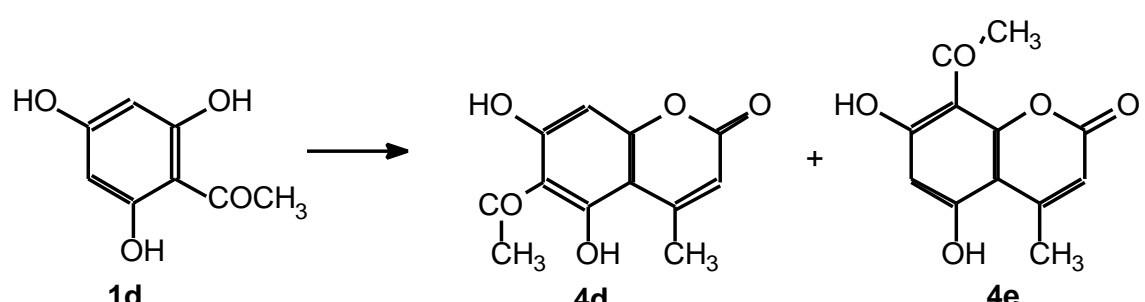<smiles>Cc1cc(=O)oc2cc(O)c3c(c12)OCC(C=O)C3=O</smiles>

$5 d_{1}$<smiles>Cc1cc(=O)oc2cc3c(c(O)c12)C(=O)C(C=O)CO3</smiles>

$5 d$ $\searrow \mathrm{DMF}, \mathrm{POCl}_{3}$<smiles>Cc1cc(=O)oc2c1c(O)cc1occ(C=O)c(=O)c12</smiles>

$5 e$

Scheme 2. 
Table 3. ${ }^{1} \mathrm{H}$ NMR - spectral data.

\begin{tabular}{|c|c|c|}
\hline compound & solvent & spectra $\delta(\mathrm{ppm})$ \\
\hline $\mathbf{1 a}$ & $\mathrm{CDCl}_{3}$ & $\begin{array}{l}12.52(1 \mathrm{H}, \mathrm{s}, \mathrm{OH}), 7.42(1 \mathrm{H}, \mathrm{s}, \mathrm{H}-6), 6.34(1 \mathrm{H}, \mathrm{s}, \mathrm{H}-3), 1.65-0.87 \\
(13 \mathrm{Hm})\end{array}$ \\
\hline $\mathbf{3 a}$ & DMSO & $\begin{array}{l}10.11(1 \mathrm{H}, \mathrm{s}, \mathrm{CHO}), 8.78(1 \mathrm{H}, \mathrm{s}, \mathrm{H}-2), 7.99(1 \mathrm{H}, \mathrm{d}, \mathrm{H}-5), 7.04-6.94 \\
(2 \mathrm{H}, \mathrm{t}, \mathrm{H}-6,8)\end{array}$ \\
\hline $3 b$ & DMSO & $\begin{array}{l}10.12(1 \mathrm{H}, \mathrm{s}, \mathrm{CHO}), 8.77 \text { (1H,s,H-2), } 7.48 \text { (1H,d,H-5), } 7.00 \\
(1 \mathrm{H}, \mathrm{d}, \mathrm{H}-6)\end{array}$ \\
\hline $3 c$ & DMSO & $\begin{array}{l}10.12(1 \mathrm{H}, \mathrm{s} . \mathrm{CHO}), 8.73(1 \mathrm{H}, \mathrm{s}, \mathrm{H}-2), 7.79(1 \mathrm{H}, \mathrm{s}, \mathrm{H}-5), 6.93 \\
(1 \mathrm{H}, \mathrm{s}, \mathrm{H}-8), 2.9(2 \mathrm{H}, \mathrm{t}), 1.30(8 \mathrm{H}, \mathrm{m}), 0.86(3 \mathrm{H}, \mathrm{t})\end{array}$ \\
\hline $4 a$ & $\mathrm{CDCl}_{3}$ & $\begin{array}{l}7.68(1 \mathrm{H}, \mathrm{d}, \mathrm{H}-5), 6.90(1 \mathrm{H}, \mathrm{d}, \mathrm{H}-6), 6.12(1 \mathrm{H}, \mathrm{s}, \mathrm{H}-3), 2.95 \\
(3 \mathrm{H}, \mathrm{s}, \mathrm{CH} \mathrm{CO}), 2.41(3 \mathrm{H}, \mathrm{s}, \mathrm{CH}), 13.54(1 \mathrm{H}, \mathrm{s}, \mathrm{OH})\end{array}$ \\
\hline $4 b$ & $\mathrm{CDCl}_{3}$ & $\begin{array}{l}7.96(1 \mathrm{H}, \mathrm{s}, \mathrm{H}-5), 6.84(1 \mathrm{H}, \mathrm{s}, \mathrm{H}-8), 6.17(1 \mathrm{H}, \mathrm{s}, \mathrm{H}-3), 2.70 \\
(3 \mathrm{H}, \mathrm{s}, \mathrm{CH} \mathrm{CO}), 2.44(3 \mathrm{H}, \mathrm{s}, \mathrm{CH}), 12.61(1 \mathrm{H}, \mathrm{s}, \mathrm{OH})\end{array}$ \\
\hline $4 c$ & $\mathrm{CDCl}_{3}$ & $\begin{array}{l}7.85(1 \mathrm{H}, \mathrm{d}, \mathrm{H}-7), 6.83(1 \mathrm{H}, \mathrm{d}, \mathrm{H}-8), 6.13(1 \mathrm{H}, \mathrm{s}, \mathrm{H}-3), 2.66 \\
(6 \mathrm{H}, \mathrm{s}, \mathrm{CHCO}), 14.07(1 \mathrm{H}, \mathrm{s}, \mathrm{OH})\end{array}$ \\
\hline 4d & $\mathrm{CDCl}_{3}$ & $\begin{array}{l}6.26(1 \mathrm{H}, \mathrm{s}, \mathrm{H}-3), 5.99(1 \mathrm{H}, \mathrm{d}, \mathrm{H}-8), 2.68(3 \mathrm{H}, \mathrm{s}, \mathrm{CH} \mathrm{CO}), 2.51 \\
(3 \mathrm{H}, \mathrm{s}, \mathrm{CH})\end{array}$ \\
\hline $4 e$ & $\mathrm{CDCl}_{3}$ & $\begin{array}{l}6.37(1 \mathrm{H}, \mathrm{s}, \mathrm{H}-3), 5.94(1 \mathrm{H}, \mathrm{s}, \mathrm{H}-6), 2.68 \text { (3H,s,CH CO), } 2.51 \\
(3 \mathrm{H}, \mathrm{s}, \mathrm{CH})\end{array}$ \\
\hline $5 a^{a}$ & DMSO & $\begin{array}{l}10.12(1 \mathrm{H}, \mathrm{s}, \mathrm{CHO}), 8.86(1 \mathrm{H}, \mathrm{s}, \mathrm{H}-2), 8.18(1 \mathrm{H}, \mathrm{d}, \mathrm{H}-10), 7.67 \\
(1 \mathrm{H}, \mathrm{d}, \mathrm{H}-9), 6.53(1 \mathrm{H}, \mathrm{s}, \mathrm{H}-7)\end{array}$ \\
\hline $5 b^{a}$ & DMSO & $\begin{array}{l}10.12(1 \mathrm{H}, \mathrm{s}, \mathrm{CHO}), 8.97(1 \mathrm{H}, \mathrm{s}, \mathrm{H}-2), 8.39(1 \mathrm{H}, \mathrm{s}, \mathrm{H}-5), 7.87 \\
(1 \mathrm{H}, \mathrm{s}, \mathrm{H}-10), 6.56(1 \mathrm{H}, \mathrm{s}, \mathrm{H}-7), 2.54(3 \mathrm{H}, \mathrm{s}, \mathrm{CH})\end{array}$ \\
\hline $5 c^{a}$ & DMSO & $\begin{array}{l}10.14(1 \mathrm{H}, \mathrm{s}, \mathrm{CHO}), 9.02(1 \mathrm{H}, \mathrm{s}, \mathrm{H}-2), 8.31(1 \mathrm{H}, \mathrm{d}, \mathrm{H}-5), 7.58 \\
(1 \mathrm{H}, \mathrm{d}, \mathrm{H}-6), 6.57(1 \mathrm{H}, \mathrm{s}, \mathrm{H}-9), 2.74(3 \mathrm{H}, \mathrm{s}, \mathrm{CH})\end{array}$ \\
\hline $5 d^{a}$ & DMSO & $\begin{array}{l}10.05(1 \mathrm{H}, \mathrm{s}, \mathrm{CHO}), 8.63(1 \mathrm{H}, \mathrm{s}, \mathrm{h}-2), 8.12(1 \mathrm{H}, \mathrm{s}, \mathrm{H}-10), 6.78 \\
(1 \mathrm{H}, \mathrm{s}, \mathrm{H}-7), 6.26(1 \mathrm{H}, \mathrm{s}, \mathrm{OH}), 2.54(3 \mathrm{H}, \mathrm{s}, \mathrm{CH})\end{array}$ \\
\hline $5 e$ & DMSO & $\begin{array}{l}10.07(1 \mathrm{H}, \mathrm{s}, \mathrm{CHO}), 9.06(1 \mathrm{H}, \mathrm{s}, \mathrm{H}-2), 7.30(1 \mathrm{H}, \mathrm{s}, \mathrm{H}-10), 6.31 \\
(1 \mathrm{H}, \mathrm{s}, \mathrm{H}-7), 2.62(3 \mathrm{H}, \mathrm{s}, \mathrm{CH})\end{array}$ \\
\hline 6 & $\mathrm{CDCl}_{3}$ & $\begin{array}{l}8.26(2 \mathrm{H}, \mathrm{t}, \mathrm{H}-2, \mathrm{CH}), 7.10-7.56(3 \mathrm{H}, \mathrm{m}, \operatorname{arom}), 4.31(2 \mathrm{H}, \mathrm{q}, \mathrm{CH}), \\
2.47(3 \mathrm{H}, \mathrm{s}, \mathrm{CH} \mathrm{COO}), 2.35(3 \mathrm{H}, \mathrm{s}, \mathrm{CH} \mathrm{CO}), 1.35(3 \mathrm{H}, \mathrm{t}, \mathrm{CH})\end{array}$ \\
\hline
\end{tabular}

${ }^{\mathrm{a}}$ spectra were recorded on a Bruker AM 300 

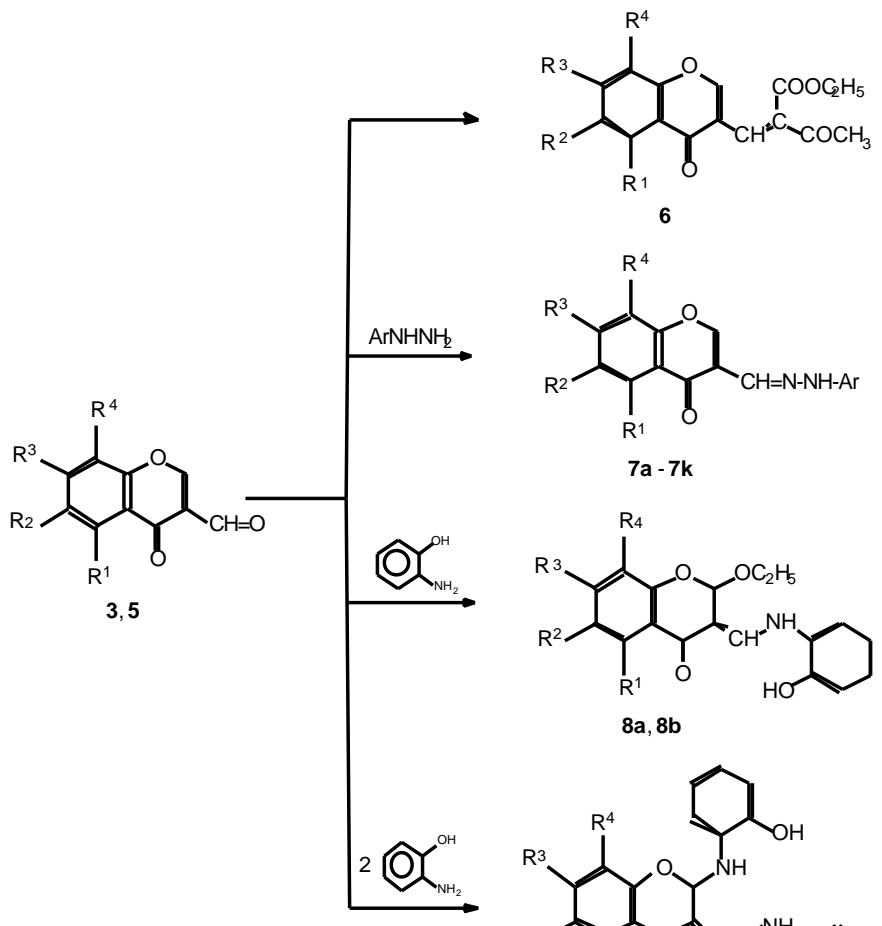

$7 a-7 k$<smiles>[R9]C1=C([R])C2OC(OC)C(CNC3CCCCC3O)C(O)C2C([R])C1[R]</smiles>

$8 \mathrm{a}, 8 \mathrm{~b}$<smiles>[R]C1=C([2H])C2C(O)C(=CNC3C=CCCC3O)C(NC3CCCCC3O)OC2C([R])C1[R]</smiles>

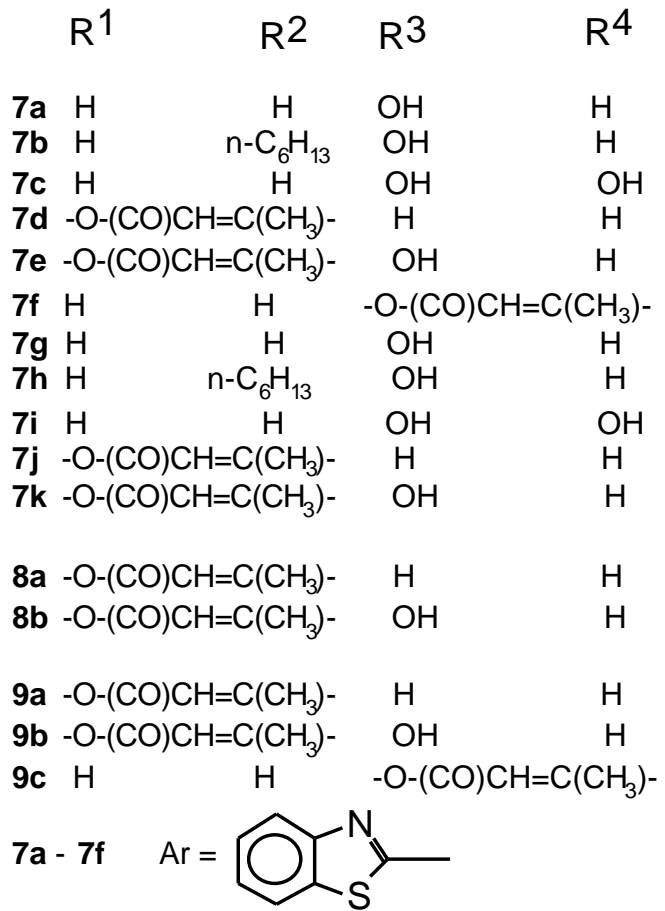

7g- 7k $\mathrm{Ar}=2,4-\left(\mathrm{NO}_{2}\right)_{2} \mathrm{C}_{6} \mathrm{H}_{3}$

Scheme 3.

\section{Experimental Section}

\section{General details}

The synthesized compounds were characterized by melting points, elemental analysis, IR and ${ }^{1} \mathrm{H}$ NMR spectra.

The melting points were determined on a Boetius apparatus and are uncorrected. The IR spectra were taken on a Specord M-80 (Zeiss) spectrophotometer in a nujol suspension.

The NMR spectra were measured on a Tesla BS 487 $(80 \mathrm{MHz})$ and Bruker AM $300 \quad(300.13 \mathrm{MHz})$ spectrometers in deuterated DMSO and $\mathrm{CHCl}_{3}$.

The synthesis of acetophenones $\mathbf{1 a}, \mathbf{1 b}, \mathbf{1 d}$ is described in papers [11 - 13] and the preparation of compounds $\mathbf{4 a}$ $\mathbf{4 e}$ in papers [14 - 16].

\section{2, 4-Dihydroxy-5-n-hexylacetophenone 1c}

4-n-Hexyl-1, 3-dihydroxybenzene (30 g, $0.15 \mathrm{~mol}$ ) was gradually added to a stirred and hot mixture $\left(120{ }^{\circ} \mathrm{C}\right)$ of glacial acetic acid $(45 \mathrm{ml})$ and anhydrous $\mathrm{ZnCl}_{2}(44.6 \mathrm{~g}$, $0.32 \mathrm{~mol})$. The mixture was refluxed for 10 minutes. After cooling the mixture was diluted with $\mathrm{HCl}(120 \mathrm{ml}$, diluted $1: 1)$ and was kept in refrigerator (12 hrs). The crystals were filtered off, washed with diluted $\mathrm{HCl}(1: 3)$ and recrystallized from methanol. Yield $25 \mathrm{~g}(72 \%)$

\section{3-Formylchromones 3, 5. General procedure}

To the dry dimethylformamide $(121 \mathrm{ml})$ in a three necked flask, $\mathrm{POCl}_{3}(0.49 \mathrm{~mol})$ was added slowly with intensive stirring at $50{ }^{\circ} \mathrm{C}$. Heating and stirring was continued for $2 \mathrm{hrs}$ at $45-55{ }^{\circ} \mathrm{C}$. The solution of 2 hydroxyacetophenone $(0.12 \mathrm{~mol})$ in DMF $(25 \mathrm{ml})$ was then slowly added under stirring at $50{ }^{\circ} \mathrm{C}$. The stirring was continued for $2 \mathrm{hrs}$ at $55-60{ }^{\circ} \mathrm{C}$. After cooling the mixture was kept over night at room temperature and diluted slowly by adding crushed ice (500 g) and stirred again for 
$6 \mathrm{hrs}$. The crystals were filtered off and recrystallized from alcohol. Yields of compounds $\mathbf{3}$ are 20 - $30 \%$, of $\mathbf{5}$ are 80 $90 \%$

3-(4-Oxo-7-acetoxy-4H-1-benzopyran-3-yl)-2-(1oxoethyl)-2-ethylpropenoate 6

A mixture of 7-hydroxy-3-formylchromone $\mathbf{3 a}(1 \mathrm{~g}, 5.3$ mmol), ethyl acetoacetate $(0.82 \mathrm{~g}, 6.3 \mathrm{mmol})$, acetic anhydride $(4.32 \mathrm{~g}, 42 \mathrm{mmol})$ and $\mathrm{K}_{2} \mathrm{CO}_{3}(0.07 \mathrm{~g}, 0.53$ mmol) was heated for $1 \mathrm{hr}$. After cooling, $30 \mathrm{ml}$ diethylether was added and the ester was allowed to crystallize over 12 hours at room temperature. A yellow solid product was filtered off and recrystalized from ethanol. Yield $56 \%$.

2-Benzothiazolylhydrazone-3-formylchromone $7 \boldsymbol{a}$ - 7f, 2, 4-dinitrophenylhydrazone-3-formylchromone $7 \boldsymbol{g}-7 \boldsymbol{k}$ and 2-ethoxy-3-(2-hydroxyphenylaminomethylene)chroman-4ones $8 \boldsymbol{a}, 8 \mathrm{~b}$

Ethanolic solutions of 3-formylchromone derivatives (1 mmol), and 2-benzotiazolhydrazine (or 2, 4dinitrophenylhydrazine, or 2-hydroxyaniline) (1 mmol) and one crystral of p-toluenesulfonic acid were mixed together and stirred for $1 \mathrm{~h}$, at $30-35^{\circ} \mathrm{C}$. The reaction mixture was then cooled to $10{ }^{\circ} \mathrm{C}$. The yellow precipitate was filtered off and recrystallized from ethanol or a mixture DMSO - ethanol. Yields about 70 - $75 \%$.

2-(2-hydroxyphenylamino)-3-(2-

hydroxyphenylaminometylene)chroman-4-ones $9 \boldsymbol{a}-\mathbf{9 c}$

The anhydrous chloroform solution $(15 \mathrm{ml})$ of 3formylchromone $(1 \mathrm{mmol})$ and 2-hydroxyaniline $(2 \mathrm{mmol})$ was stirred for 30 minutes at $50{ }^{\circ} \mathrm{C}$. After cooling the mixture petroleum ether was added to form a precipitate. The product was filtered off. Toluene was used for recrystalization. Yields $50-58 \%$.

Acknowledgements: The authors would like to thank Dr. E. Greiplova for elemental analysis, Mgr. J. Prokes for ${ }^{1} \mathrm{H}$ NMR measurements (80 MHz), Dr. A. Perjessy for IR spectral measurements. Financial support for this research from the Slovak Agency (grant No. 1/5058/98) is gratefully acknowledged.

\section{References}

1. El-Shaer, H. M.; Zahradnik, P.; Lacova M.; Matulova, M. Collect. Czech. Chem. Commun. 1994, 59, 1673.

2. El-Shaaer, H.M.; Lacova, M.; Odlerova, Z.; Furdik, M. Chem. Papers 1994, 59, 1673.

3. El-Schaaer, H.M.; Perjessy, A.; Zahradnik, P.; Lacova M.; Sustekova Z. Monatsh. Chem. 1993, 124, 539.

4. Gasparova, R.; Lacova, M. Collect. Czech. Chem. Commun. 1995, 60, 1178.

5. Lacova, M.; Stankovicova, H.; Odlerova, Z. Il farmaco 1995, 50, 885.

6. Kralova, K.; Sersen, F.; Lacova, M.; Stankovicova, H. Biol. Plant. 1995, 38, 397.

7. El-Schaaer, H.M.; Foltinova, P.; Lacova, M.; Chovancova, J.; Stankovicova, H. Il - farmaco (in press).

8. Nohara, A.; Umetani ,T.; Sanno, Y. Tetrahedron 1974, 30, 3553.

9. Nohara, A.; Umetani, T.; Sanno, Y. Tetrahedron Lett. 1973, (22) 1995-8.

10. Dewar, M. J. S.; Zoebisch, E. G.; Healy, E. F.; Stewart, J. J. P. J. Am. Chem. Soc., 1985, 57, 3698.

11. Brown, R.C.; Harard, R. U.S. 1980, 4238, 606 ; Chem. Abstr. 1981, 94p, 156755.

12. Klutchko, S.; Kaniansky, D. U.S. 1977, 4008, 232; Chem. Abstr. 1977, 87p, 5808.

13. Cooper, R. S. Org. Synth. Coll. Vol. III.761, John Wiley and Sons, New York 1967.

14. Desai, R.D.; Ekhlas, M. Proc Indian Acad SCi (A), 1938, 567, Chem. Abstr. 1939, 33, 3356.

15. Sethna, S.M.; Shah ,R.C. J. Chem. Soc. 1938, 228.

16. Hoesch, K. Ber. 1942, 48, 1125.

Samples Availability: Samples are available from MDPI and the authors. 